\title{
Advantages of Copper Backhauling for G.Fast Nodes
}

\author{
Frank Phillipson and Rob F. M. van den Brink
}

\begin{abstract}
A next step in bringing high bandwidth over an existing copper infrastructure is Fibre to the Curb, using G.Fast, which prevents a huge investment compared to a full fibre roll out. To save additional costs in this roll out, the existing copper can be reused as backhaul for the new active point of G.Fast, where currently mostly a fibre backhaul is considered. This paper presents the advantages of copper backhauling for G.Fast nodes and studies the planning and expected bandwidth coverage of this Copper Backhaul solution in two cases. These cases show that this solution is a logical next step in the network migration, offering cost saving, flexibility and the needed bandwidth gain for the consumers.
\end{abstract}

Index Terms-Access network planning, next generation broadband, G.Fast performance.

\section{INTRODUCTION}

In the near future new internet services will be so demanding in bit rate that they easily consume a band-width of hundreds of $\mathrm{Mb} / \mathrm{s}$. These services probably include multiple UHD, $4 \mathrm{~K}$ or even $8 \mathrm{~K}$ video channels simultaneously. To deliver these services to consumer's homes the use of fibre will be inevitable but this does not necessarily mean that fibre is to be deployed all the way to a point into the home, Full Fibre to the Home (Full FttH). A known alternative is bringing fibre up or near to the home, reusing existing copper cables for the last 200-300 meter. The copper technology that is required for such a Fibre to the Curb (FttCurb) or Hybrid FttH solution with sufficient bandwidth is currently developed and is named G.Fast. First results of this development make it plausible that Hybrid FttH using G.Fast is technically feasible up to $1 \mathrm{~Gb} / \mathrm{s}$ [1]. The International Telecommunication Union is carrying on a standardisation activity for the G.fast solution as part of the so-called fourth generation broadband system architectures [2]. For the roll out of this Hybrid FttH solution, new active nodes should be installed and connected by a fibre backhaul. This application of G.Fast has some huge benefits: Hybrid FttH is cheaper than Full FttH, even if Full FttH is the long term solution [3], [4] and is much faster to roll out to provide customers with the demanded bandwidth.

In this paper we propose the use of existing copper as backhaul for the G.Fast nodes instead of new fibre cables and present the advantages of this approach. Most of earlier work on G.Fast focuses on the performance of G.Fast solely or connected by a fibre network [1], [5], [6] and on the planning of the G.Fast solution [7], also based on a fibre connection to the new G.Fast node. In [8] already a Copper Backhaul is

Manuscript received May 9, 2015; revised November 14, 2015.

The authors are with the Netherlands Organisation of Applied Research (TNO), POBox 96800, 2509 JE the Hague, Holland (e-mail: frank.phillipson@tno.nl). presented as alternative for the fibre connection for the G.Fast node, based on ideas in [9]. The main benefits of this approach is not presented in detail there.

A G.Fast solution based on a Copper Backhaul in our opinion is:

- easy scalable with market demands;

- cost effective;

- simple to implement.

Starting with a G.Fast solution that is based on a Copper Backhaul allows for gradual migration: grow of capacity can be proportional with market demand. It has the possibility of reuse of existing copper and vectored VDSL equipment, and asks only for the addition of 'super bonding' ${ }^{1}$ to existing VDSL. In this phase only new active nodes should be installed, no digging to place new fibre cables is needed. When market demand increases some Copper Backhauls are decreased in length, introducing new nodes as starting point of the Copper Backhaul, which have to be connected by fibre cable. This can be repeated, in theory, until the classical Hybrid or even Full FTTH has been reached. This approach postpones the (huge) investment costs of digging fibre connections and the gradual grow can even prevent overcapacity in the network. An interesting side effect of this solution is that the equipment of the G.Fast node could also be powered by this backhaul copper solution. Powering of this node is still a point of research.

In the remainder of this paper first the starting position of the copper network, the optimal planning concept of the G.Fast network and a more practical migration approach are presented. Next the expected bandwidth coverage of the roll out of this G.Fast variant in the Dutch cities Amsterdam and Den Haag (The Hague) is shown, answering the question: 'if the Copper Backhaul is used without any additional digging, starting at the Central Office, what is the bandwidth that the consumers can expect'. We show in Section III that even in this case the bandwidth cover-age is promising and we show the effect of gradually decreasing the length of the Copper Backhaul.

\section{Optimal PlanNing AND Migration APPROACH}

The Netherlands have a very dense copper telecommunication infrastructure. When we look in more detail to this part of the copper network we see a situation as shown in Fig. 1. This is a typical situation in the last mile of the Dutch copper network: a heavily branched network, with at the right side a Cabinet. Bundles of copper wires are leaving the Cabinet and split towards distribution links. The houses are connected to this distribution link.

\footnotetext{
${ }^{1}$ Bonding of hundreds of copper pairs.
} 


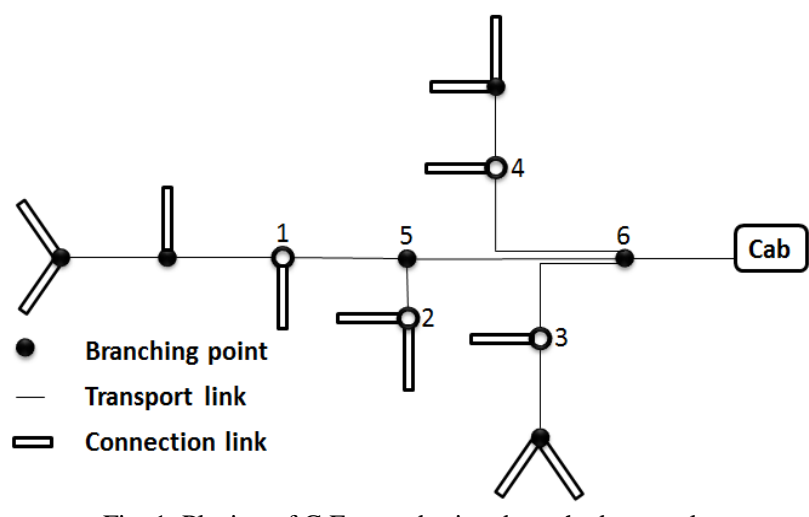

Fig. 1. Placing of G.Fast nodes in a branched network.

In this network topology new network nodes have to be placed for the G.Fast technology on a distance of 300m at maximum. To do this, possible locations for these network nodes have to be determined. Logical places for these nodes are the dots in the figure, the branching points of the network. If it is known which houses are connected to these locations at which distance, one should decide which locations will be used and how they are connected to a fibre node in the most economical way. This process is described in [7]. A possible result is depicted in Fig. 1. Here branching points 1, 2, 3 and 4 are chosen as G.Fast nodes.

If the Copper Backhaul is used as feeder for the G.Fast nodes less fibre has to be installed, saving additional digging and installation costs. The longer the Copper Backhaul, the lesser the fibre roll out, but also the lesser the capacity of the G.fast node. The fibre connection is assumed to have a higher capacity than the Copper Backhaul. Given a desired capacity of the Backhaul, based on the bandwidth per connection and a overbooking factor [10], the potential starting locations of the Backhaul can be determined. A general understanding of overbooking is to have the sum of the allocated bandwidth of flows on a link exceed the links physical bandwidth so as to achieve multiplexing gain. When five flows have a maximal bitrate of $200 \mathrm{Mb}$ and the physical link has a capacity of 500 $\mathrm{Mb}$, the overbooking factor equals $1000 / 500=2$.

In the example of Fig. 1 the place the Backhaul can start could be 5, 6 and the Cabinet. In the case of using 5 or 6 as starting point of the Backhaul, still a fibre connection has to be installed. However this fibre connection is of a shorter distance than when all the G.Fast nodes has to be connected by fibre. Using the Cabinet would lead to no fibre installation, but will give the longest distance of the Copper Backhaul and thus the lowest bandwidth. Based on the schematic approach of [7] the problem of which location (or locations) to use as starting point for the Copper Backhaul optimally is a capacitated Facility Location Problem that can be solved by the heuristic described in [11]. In this way the total costs (including connection and equipment) of the new nodes in minimized, using a dimensioning rule per backhaul $(\mathrm{BH})$, based on the tolerated overbooking:

$x=$ number of users

$d=$ maximal demand of user (in $\mathrm{Mb}$ )

$f=$ overbooking factor in backhaul

$C=$ capacity per copper wire in $\mathrm{BH}$ (in $\mathrm{Mb}$ )

$n=$ number of copper wires in $\mathrm{BH}$

$C_{d}=$ capacity demand in $\mathrm{BH}$ (in $\mathrm{Mb}$ )
$C_{s}=$ capacity supplied in $\mathrm{BH}$ (in $\mathrm{Mb}$ )

Then the capacity constraints are:

$$
\begin{gathered}
C_{s} \geq C_{d} \\
n . C_{\geq} d \cdot x / f
\end{gathered}
$$

In this way the desired bandwidth, given an assumed overbooking factor leading to a certain performance, at minimum costs is realized in one step. How-ever, in practice a more gradual roll out is expected. In [3] a migration path from a full copper access network to a full FttH access network is described. However, the step from FttN (Fibre to the Node) or FttCab (Fibre to the Cabinet, both realized by VDSL: Very-high-bitrate Digital Subscriber Line), to G.Fast, in the aforementioned study done in one step, can itself be based on a migration path. Then in the first step all the G.Fast nodes are installed and are all fed by a Copper Backhaul, starting at the Cabinet. Then gradually new points can be selected as starting point of the Copper Backhaul, based on the approach described above, shortening the copper distance where the capacity of the Backhaul is insufficient. This may converge to a situation where all G.Fast nodes are connected with fibre cables themselves. The bandwidth coverage in terms of bandwidth per end-user of this migration approach is presented in the next section.

\section{BANDWIDTH COVERAGE}

In this section the bandwidth coverage of the migration approach is calculated. In the previous section is described how the position of the G.Fast nodes and the starting points of the Copper Backhaul is calculated, such that the costs are minimized and a certain bandwidth, based on overbooking rules, is guaranteed. Using the method of [11] this has been done for some cases. We now look at the practical bandwidth coverage of such a planning realization.

The introduction of the Copper Backhaul means introducing a new potential bottleneck in the network that is shared among multiple users. For the band-width per user, three values can then be distinguished:

- Guaranteed rate (GR): the bandwidth the user gets if all users are asking their maximum value; this is the minimum of the G.fast speed and the proportionate share in the shared link, where the latter is the Copper Backhaul capacity divided by the number of connections.

- Peak rate (PR): the bandwidth the user gets if he is the only user; this is the minimum of the G.fast speed and the capacity of the shared link.

- Perceived rate: the bandwidth the user gets on average: this is the minimum of the G.fast speed and the experienced share in the shared link.

If user $i$, based on his distance to the G.Fast node, has access speed $S_{i}$ (between the house and the G.Fast node), the capacity of the Copper Backhaul is $\mathrm{C}$ and the number of users equals n, then: $G R=\min \left(S_{i}, C / n\right)$ and $P R=\min \left(S_{i}, C\right)$.

Suppose a household has a access speed of $400 \mathrm{Mb}$ and the shared link has a capacity of $2.5 \mathrm{~Gb}$ with 20 connections. Here the chosen overbooking factor by the operator is thus 3.2. 
Then the values are:

- $\mathrm{GR}=\min (400,2500 / 20)=125 \mathrm{Mb}$;

- $\mathrm{PR}=\min (400,2500)=400 \mathrm{Mb}$;

- The perceived rate lies in between and is highly dependent on the behaviour of users that we cannot predict. But we can, given the load on the shared link, translate this load back to the expected perceived rate.

To test the approach first for a small neighbourhood in Den Haag these values are calculated based on real houses and distances. This neighbourhood consists of 1300 houses. The used relation between bandwidth of VDSL and G.Fast and distance is depicted in Table I and are based on measurements (VDSL) and expectation (G.Fast) of TNO. The values of G.Fast are realized when also VDSL has to be provided to clients, resulting in a less effective G.Fast connection. This is the case where the regulator demands to provide VDSL-access for other parties. G.Fast Alone represents the bandwidth if only G.Fast is offered. G.Fast access speeds are only depicted for distances up to 300 meter.

Fig. 2 shows the result of this analysis. Here the distribution of different bandwidth values over the connections, realized by choosing a high overbooking factor, are shown. This high overbooking factor in the model results in Copper Backhauls originating in the Cabinet. The figure shows the probability that a connection (user) gets more than a certain bandwidth in a certain scenario. In the case that VDSL from the Cabinet (or Node) is offered, where all Cabinets are connected with a fibre network, $90 \%$ of the connections get more than $30 \mathrm{Mb}$, $50 \%$ of connections get more than $70 \mathrm{Mb}$ and $10 \%$ has more than $110 \mathrm{Mb}$. This is the line 'FttN/VDSL' in the figure, the line that is the closest to the y-axis. If G.fast, connected with a Copper Backhaul to these Cabinets ${ }^{2}$, is introduced, the distribution shifts to the next line, 'Guaranteed', for the distribution of the Guaranteed rate. Now $90 \%$ of the connections get over $120 \mathrm{Mb}$ as guaranteed value, $50 \%$ more than $220 \mathrm{Mb}$ and $10 \%$ more than $490 \mathrm{Mb}$. The peak values here are $440 \mathrm{Mb}, 600 \mathrm{Mb}$ and $620 \mathrm{Mb}$, respectively, close to the bandwidth offered by the G.Fast part of the connection only. To summarize this: $90 \%$ of the connections has in the VDSL case more than $30 \mathrm{Mb}$ and will have a Guaranteed rate of more than $120 \mathrm{Mb}$ with a peak rate of more than $400 \mathrm{Mb}$ when G.Fast, using a Copper Backhaul, is offered. This is already a significant improvement of the bandwidth.

\begin{tabular}{llll}
\multicolumn{4}{c}{ TABLE I: BANDWIDTH IN MB FOR VDSL AND G.FAST } \\
\hline \hline Distance (m) & VDSL & G.Fast & G.Fast alone \\
\hline 50 & 125 & 610 & 810 \\
100 & 125 & 550 & 740 \\
150 & 125 & 460 & 690 \\
200 & 85 & 320 & 490 \\
250 & 70 & 200 & 380 \\
300 & 60 & 120 & 290 \\
400 & 45 & - & - \\
800 & 35 & - & - \\
1300 & 20 & - & - \\
2000 & 8 & - & - \\
\hline \hline
\end{tabular}

Gradually selecting new points smartly as starting point of the Copper Backhaul and connecting them with fibre gives

\footnotetext{
${ }^{2}$ Using the G.Fast variant of Fig. 3.
}

more bandwidth on bottleneck locations. The result of 11 new starting points ${ }^{3}$ and connecting them with $1.25 \mathrm{~km}^{4}$ fibre is shown in Fig. 3. Now $90 \%$ get over $210 \mathrm{Mb}$ (was $120 \mathrm{Mb}$ ) as guaranteed value, $50 \%$ more than $310 \mathrm{Mb}(220 \mathrm{Mb})$ and $10 \%$ more than $520 \mathrm{Mb}(490 \mathrm{Mb})$. Connecting all the G.Fast nodes would require $3.65 \mathrm{~km}$ of fibre. Taking a total cost of 35 euro per meter for installing new fibre cable (including digging, the cable, refilling and repaving) the initial situation, using the Copper Backhaul all the way to the cabinet, saves 127,750 euro. This is almost 100 euro per end-user. The cost of the G.Fast connection with fibre backhaul in this case we estimate on 300 euro. The estimated cost of a full-fibre solution is about 970 Euro. The effect on costs of active equipment asks for further study.

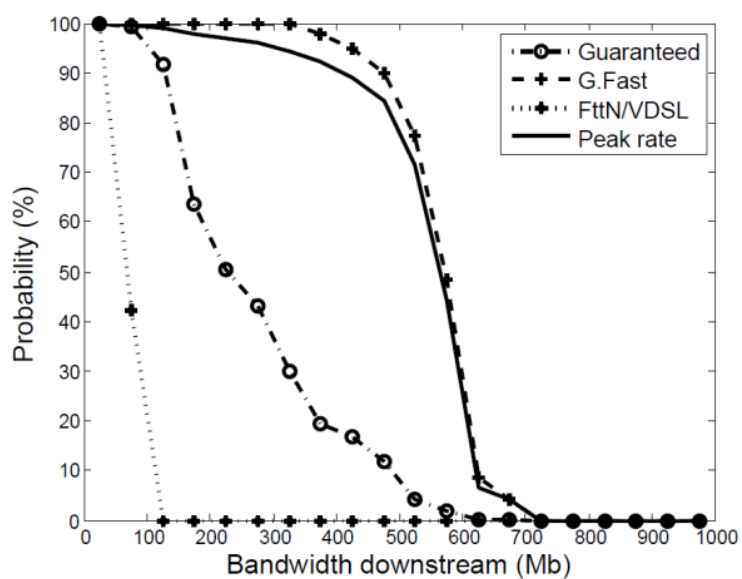

Fig. 2. Probability of bandwidth higher than value $x$.

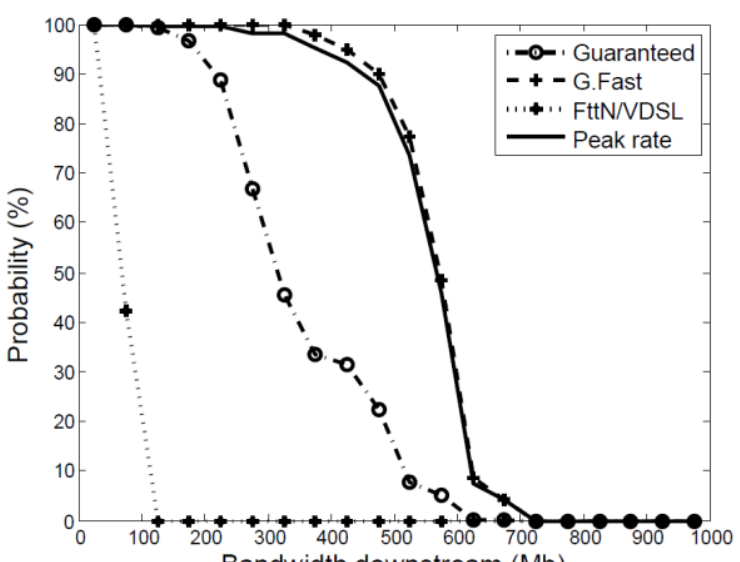

Fig. 3. Probability of bandwidth higher than value $x$ : introducing extra nodes.

If we repeat this exercise for the city of Den Haag as a whole (208,000 connections), again using the Copper Backhaul all the way to the cabinets, the results are as depicted in Fig. 4. In Den Haag 90\% get over $40 \mathrm{Mb}$ as guaranteed value, $50 \%$ more than $280 \mathrm{Mb}$ and $10 \%$ more than $520 \mathrm{Mb}$. In comparison to the single neighbourhood earlier, the figure starts closer to the $y$-axis, resulting in smaller values as minimum for the $90 \%$ case, $40 \mathrm{Mb}$ against $120 \mathrm{Mb}$. In Fig. 4 also the potential of G.Fast is shown, when G.Fast is the only technique used, called 'G.Fast Alone' earlier.

\footnotetext{
${ }^{3}$ This situation is created using a desired bandwidth of $1000 \mathrm{Mb}$ and an overbooking factor of 6 .
} 


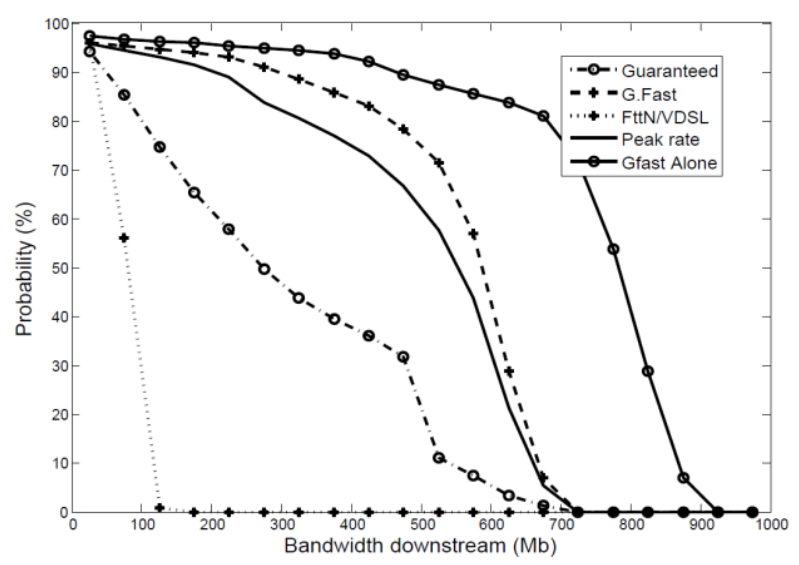

Fig. 4. Probability of bandwidth higher than value $x$ : Den Haag.

Introducing 500 new nodes, similar to the previous case where 11 new nodes were used to give more band-width on bottleneck locations, will lift the bandwidth coverage for the city of Den Haag to: $90 \%$ get over $90 \mathrm{Mb}$ as guaranteed value, $50 \%$ more than $290 \mathrm{Mb}$ and $10 \%$ more than $520 \mathrm{Mb}$.

In the case of the city of Amsterdam (376,000 connection), as shown in Fig. 5, the guaranteed value lies closer to the peak rate than in the other cases. Here, without any extra fibre, $90 \%$ get over $130 \mathrm{Mb}, 50 \%$ over $500 \mathrm{Mb}$ and $10 \%$ over $610 \mathrm{Mb}$. This confirms the results of [7] where already was shown that the copper length from the end-user to the Cabinets are relatively short in Amsterdam, resulting in a relatively cheap introduction of G.Fast. Now also is clear that for introduction of G.Fast in Amsterdam the installing of fibre is hardly needed. Only in the case no VDSL is offered and G.Fast can perform on its maximum speed, shorter Copper Backhaul distances are desirable. When G.Fast is offered, using Copper Backhaul, this will give guaranteed rates very close to the G.Fast access maximum values.

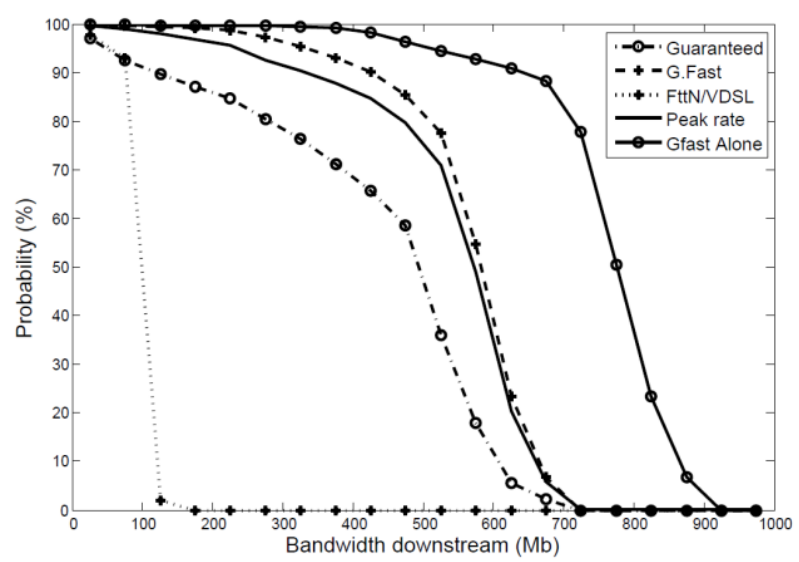

Fig. 5. Probability of bandwidth higher than value $x$ : Amsterdam.

\section{CONCLUSIONS}

In this paper the option of a Copper Backhaul to connect the G.Fast active nodes is studied. This option is a lot cheaper than installing new fibre cables for this connection. This Copper Backhaul can be realized starting at the Cabinet or at new active points who have to be connected by a fibre

\footnotetext{
${ }^{4}$ The fibre distances are worst case: a tree structure following the original copper lines.
}

network. The first option is cheaper but has a lower bandwidth. In this paper the optimal planning procedure of this Copper Backhaul option is presented, leading to the possibility to gradually introduce the new active points as starting point for the Copper Backhaul. Starting with the Cabinets as starting point for the Copper Backhaul gives already an increase in guaranteed bandwidth for the end users and is a valuable start position for an operator when rolling out G.Fast at minimal cost.

\section{REFERENCES}

[1] M. Timmers, M. Guenach, C. Nuzman, and J. Maes, "G.Fast: Evolving the copper access network," IEEE Communications Magazine, vol. 51, no. 8, pp. 74-79, 2013.

[2] R. V. D. Brink, "Enabling 4GBB via the last copper drop of a hybrid FttH deployment," Broadband Journal of the SCTE, vol. 33, no. 2, pp. 40-46, 2011.

[3] F. Phillipson, "A cost effective topology migration path towards fibre," in Proc. the 3rd International Conference on Information Communication and Management, Paris, France, 2013, pp. 24-31.

[4] J. R. Schneir and Y. Xiong, "Economic aspects of fibre to the distribution point with G. Fast," Telecommunications Policy, 2014.

[5] M. Jensen and J. M. G. Lopez, "Upper bound performance estimation for copper based broadband access," in Proc. 20th Telecommunications Forum, Belgrade, Serbia, 2012, pp. 280-283.

[6] A. Umek, "Next generation copper broadband access," in Proc. in 2nd Int. Conference on Information Society Technology, 2012, p. 214.

[7] F. Phillipson, "Planning of fibre to the curb using G.Fast in multiple rollout scenarios," in Proc. the 3rd International Conference on Information Communication and Management, pp. 42-49.

[8] P. Odling, T. Magesacher, S. Host, P. O. Borjesson, M. Berg, and E. Areizaga, "The fourth generation broadband concept," IEEE Communications Magazine, vol. 47, no. 1, pp. 62-69, 2009.

[9] J. M. Cioffi, S. Jagannathan, M. Mohseni, and G. Ginis, "Cupon: The copper alternative to pon $100 \mathrm{gb} / \mathrm{s}$ dsl networks," IEEE Communications Magazine, vol. 45, no. 6, pp. 132-139, 2007.

[10] J. Liu, X. Jiang, and S. Horiguchi, "Opportunistic link overbooking for resource efficiency under per-flow service guarantee," IEEE Transactions on Communications, vol. 58, no. 6, pp. 1769-1781, 2010.

[11] F. Phillipson, "Fast roll-out of fibre to the cabinet: Practical approach for activation of cabinets," in Proc. 2014 19th European Conference on Networks and Optical Communications, 2014, pp. 102-107.

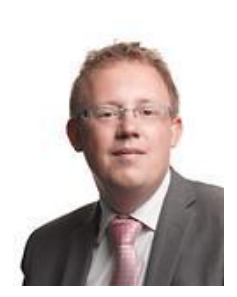

Frank Phillipson graduated in econometrics and operations research at VU University Amsterdam in 1996. In 1998 he received a 'professional doctorate in engineering' from the Delft University of Technology in mathematical design engineering. From 1998 until 2003, he was employed at KPN (Royal Dutch Telecom). Frank is currently working as a senior scientist in the Department of Performance of Networks and Systems at TNO, working on optimization and cost models for telecommunication and electricity network capacity management. In 2014, Frank received his PhD degree in applied mathematics from VU University Amsterdam on planning of infrastructure networks.

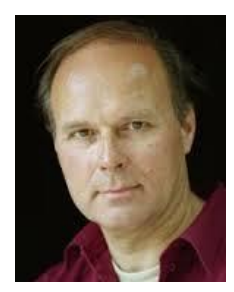

Rob F. M. van den Brink graduated in electronics from Delft University in 1984, and received his $\mathrm{PhD}$ degree in 1994. He works as a senior scientist within TNO on broadband access networks. Since 1996, he has played a very prominent role in DSL standardisation in Europe (ETSI, FSAN), has written more than 100 technical contributions to ETSI and took the lead within ETSI-TM6 in identify-ing/defining cable models, test loops, noise models, performance tests and spectral management. He has also been the rapporteur/editor for ETSI since 1999 (on Spectral Management: TR 101 830), a board member of the MUSE consortium (2004-2008, www.ist-muse.org) and work package leader within the Celtic 4GBB Consortium since 2009 (www.4gbb.eu). 